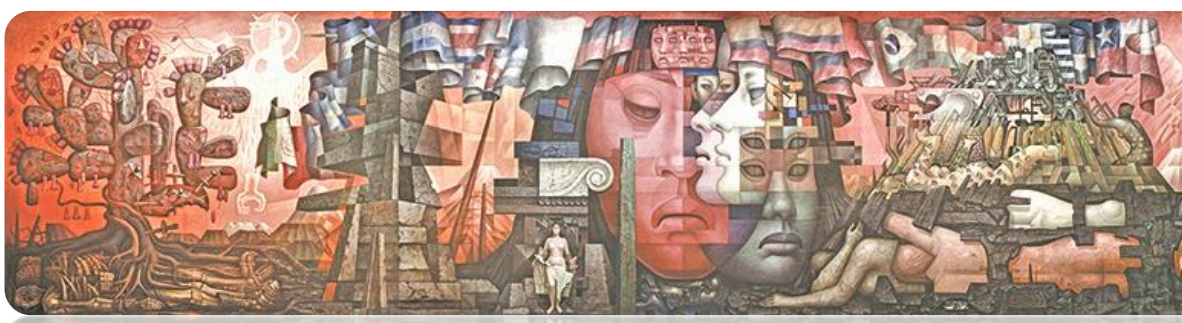

Rev. Hist., N²8, vol. 1, Julio-Diciembre 2021: 12-16

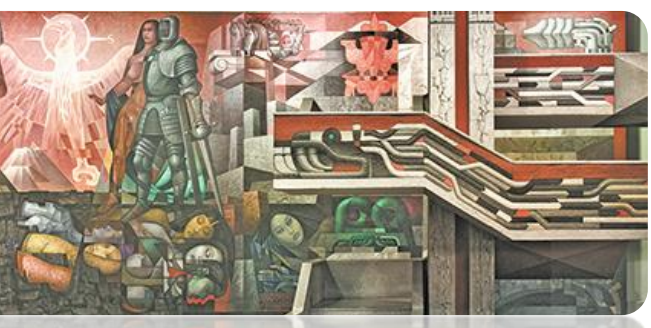

ISSN 0717-8832

https://doi.org/10.29393/RH28-23OSEC10023

\title{
Presentación dossier:
}

\section{O’Higgins-San Martín. Chile y Perú. Proyectos y proyecciones históricas e historiográficas}

\author{
O'Higgins-San Martín. Chile and Peru. Historical and historiographic projects and \\ projections
}

\begin{abstract}
A 200 años del desembarco de la expedición libertadora al mando del General José de San Martín en el Perú, en una interesante entrevista, el historiador peruano José de la Puente Brunke pensaba que para entender el hecho mismo del 8 de setiembre, debería tenerse en cuenta todas las dificultades y complejidades que esa expedición implicó. Señalaba que, en las Provincias Unidas del Río de la Plata, la idea dominante fue el luchar contra el virrey a partir del norte argentino hacia el Alto Perú, mientras que San Martín, con mucha insistencia, planteaba la idea de hacerlo por mar desde Chile, lo cual le supuso una serie de rupturas con los líderes de las Provincias Unidas al punto de que el apoyo que recibió fue el del director supremo de Chile, Bernardo O'Higgins. Ante la pregunta si O'Higgins nombró a la expedición o si fue San Martín quien lo convenció, la respuesta de Puente Brunke es muy asertiva:
\end{abstract}

“Los dos lo tenían muy claro. O'Higgins y todos, en el fondo, veían que era requisito fundamental lograr la derrota del gran poder virreinal en América del Sur que era Lima. El tema estaba en el cómo. Entonces, como director supremo de Chile, O'Higgins es el que respalda. Otra idea que me parece importante es entender la expedición libertadora en el contexto del carácter hispanoamericano de la independencia. Finalmente, la independencia no es un fenómeno peruano, ni chileno ni argentino sino continental... [Agrega, más adelante]: En el fondo (San Martín) rompe con las Provincias Unidas para luego entrar directamente a trabajar coordinadamente con el director supremo de Chile, quien incluso le 
da una serie de instrucciones. Hay polémica sobre si San Martín siguió o no las instrucciones de O’Higgins, porque por ejemplo se nombra protector del Perú cuando la idea original había sido que él nombrara para dirigir políticamente al Perú a un peruano y que él se dedicara, más bien, a la parte militar. Uno de los temas que más se ha criticado a San Martín, además del tema monárquico, es que él estableciera el protectorado y que se autodenomine protector del Perú" ${ }^{1}$.

Tres problemas que se adelantan: el reconocimiento al rol jugado por Bernardo O'Higgins en el proceso de la Independencia del Perú; en segundo lugar, el hecho de que independientemente de las acciones militares concretas desarrolladas por los próceres de la emancipación, ésta necesitó igualmente de un ideario y un proyecto americano en el cual O'Higgins no estuvo ausente $y$, en tercer lugar, que la organización del plan marítimo de expedición al Perú, en términos de las relaciones personales entre don Bernardo y don José de San Martín, fue en mucho superior a los diferentes criterios y principios declarados y defendidos por ambos respecto al régimen de gobierno a implantar: republicanismo o monarquía, respectivamente.

Poco antes de la irrupción de la pandemia Covid-19, con el patrocinio de la Facultad de Ciencias Sociales de la Universidad Nacional Mayor de San Marcos, nos reunimos en Lima un grupo de historiadores chilenos y peruanos para dialogar sobre los roles jugados por O'Higgins y San Martín en la Independencia del Perú. Nadie dudaba, ni duda, respecto al reconocimiento que se le otorga en dicho país al General San Martín y, aun cuando no se desconoce el papel asumido por O'Higgins, la propia historia y la historiografía peruana y latinoamericana le reducen su participación por cuestiones de acción militar de primera línea o por los desarrollos seguidos por la historia chileno-peruana a lo largo del siglo XIX. Por ejemplo, siguiendo lo publicado por Mijail Palacios, ya anotado, Carmen Mc Evoy señala que, hay

"testimonios de muchas mujeres en Valparaíso con gran emoción despidiendo a hijos y esposos. Incluso, se organizaron una serie de festejos porque de alguna manera era una expedición que iba a asegurar la independencia de Chile. Cuando se celebró el centenario de la expedición, el presidente Leguía y todo su gobierno se trasladó a Pisco. No sé si el presidente Vizcarra lo hará. Pero bueno, en los discursos políticos de esa ceremonia en Pisco todos fueron elogios a Argentina, prácticamente no se menciona a Chile, porque en 1920 el Perú estaba en lo más álgido del conflicto diplomático con Chile por el tema de las provincias cautivas de Tacna y Arica. En 1920 nadie en el Perú valoró ni puso en relieve lo que era

1 Mijail Palacios, José de San Martín y el desembarco de la expedición libertadora, mpalacios@peru21.com. Actualizado el 7 de septiembre de 2020. 
cierto: el apoyo fundamental que tuvo San Martín fue del gobierno chileno. Y no hay por qué discutirlo porque es prueba del carácter hispanoamericano de la independencia"².

Existe un problema de fondo que se ha exteriorizado en tiempos de pandemia y que se ha nutrido de nuevos movimientos contestatarios a la historia, al pasado y al papel de lo que se ha venido recordando y respetando como hombres que transformaron la historia o aquellos que construyeron Estados nacionales. Movimientos como Black Lives Matter han puesto en un primer plano los problemas de racismo, colonización, explotación, etc. y ello ha llevado a que, tanto en Estados Unidos como en Europa, hayan caído importantes monumentos y con ello las figuras que rememoraban. ¿Qué hacer con el pasado? El distinguido académico español José Álvarez Junco, con Antonio Cazorla, escribieron en octubre del 2020 una columna de opinión en la cual señalaban que, "Las sociedades humanas tienden a presentar su propio pasado con orgullo, como una sucesión de hechos gloriosos en los que no hay nada de lo que hoy deban avergonzarse. Esa es la historia que se enseña en las escuelas, la que se difunde para consumo de masas, la que se repite en rituales y conmemoraciones públicas. La realidad, sin embargo, es más compleja" ${ }^{3}$. Aun cuando ellos se refieren básicamente a las historias nacionales de Europa que olvidan hechos violentos en sociedades con las cuales han estado en conflicto, situaciones que escapan de las lecciones de historia que se enseñan a los niños en las escuelas, no desestiman que la celebración de efemérides históricas tiene, pues, como principal función el cultivo del ego nacional, todo lo cual, en el caso de España, se aleja del conocimiento histórico riguroso tal como lo hacen los líderes que, desde el otro lado del charco, se erigen en herederos y defensores de unas víctimas con las que apenas les une vínculo alguno y concluyen en que el interés de los políticos y corifeos habituales no debieran repetir impunemente aquello que los historiadores profesionales saben son falsas o distorsionadas y citan el caso de Alemania que, en las últimas décadas, «ha hecho un gran esfuerzo público por educar a la población, no sólo sobre el horror nazi, sino también sobre la participación en el mismo del conjunto de la sociedad... Allí han asumido que los males del pasado se combaten enseñando a los ciudadanos una buena historia» ${ }^{4}$.

La negación de parte del pasado, especialmente en lo que se refiere a los héroes nacionales, fundadores o padres de la Patria, no ha escapado a Chile y, por cierto, O'Higgins también ha entrado en la lista del revisionismo histórico. Con fundamentos o sin ellos, para muchos no sólo se trata de refundar el país en presente, sino también hacerlo con la "invención" de una nueva historia desde el pasado. Los fracasos de la República advertidos en el presente se advierten y juzgan a partir de sus inicios matrices y se soslaya el largo período intermedio, dos siglos, en

\footnotetext{
2 Ídem.

3 José Alvarez Junco y Antonio Cazorla, ¿Qué hacer con el Pasado?, El País, Madrid, 23 de octubre 2020.

4 Ídem.
} 
que la sociedad igualmente ha sido responsable de sus propias trayectorias y de la larga lista de proyectos incumplidos. Es cierto que nuestras miradas respecto a la historia surgen en gran medida respecto a nuestras experiencias del hoy, pero también es cierto que el pasado debe igualmente juzgarse en términos de sus propias circunstancias para tratar de entenderlo más cabalmente, aun cuando no estemos plenamente de acuerdo con lo sucedido entonces. A nivel latinoamericano, las figuras de Simón Bolívar y el propio José de San Martín deberían desaparecer de nuestras galerías o panteones históricos si los evaluamos sólo por sus ideas monárquicas, pero ello no fue toda su historia.

Así entonces, nuestro propósito en Lima fue hacer una buena historia y tratar de traducir en mejores sentidos el pensamiento, acción y esfuerzos acometidos por don Bernardo y por don José en pos de alcanzar independencias nacionales y la independencia americana. Especialmente, en cuanto a O'Higgins, se trató de trazar su formación y madurez política considerando la mayor disponibilidad de datos disponibles (no dudamos que existen muchos más) y con ello resituarle en aspectos específicos mayores.

En el presente dossier, se consideran cinco de las comunicaciones presentadas en dicho Encuentro en la Universidad Nacional Mayor de San Marcos. En primer lugar, se ofrecen detalladamente aspectos importantes en la formación doctrinaria e ideológica de O'Higgins en diversos momentos de su vida y circunstancias que le llevaron a la primera magistratura nacional y desde allí proyectar sus objetivos independentistas de Chile, del Perú y de la América Latina. En segundo lugar, se considera igualmente, el espacio geo - estratégico desde donde se materializa el embarque de la Expedición Libertadora al Perú: Valparaíso, puerto que siguiendo su propia trayectoria pasa de ser de un lugar propio de América a ser el centro del crecimiento económico de Chile y con ello competencia del Callao que durante los siglos coloniales no había tenido grandes límites a su hegemonía en el Pacífico Sur. La competencia distorsiona las relaciones chileno-peruanas y lleva a un conflicto creciente que culmina con la Guerra del Pacífico. Ello, igualmente, distorsiona el significativo rol desempeñado por O'Higgins en la independencia peruana.

En tercer lugar, están aspectos significativos de la vida de don Bernardo en su exilio en Lima. No sólo lo que aparentemente fue una especie de tranquila vida de hacendado gracias a los beneficios otorgados por el gobierno peruano en señal de su reconocimiento a los esfuerzos desplegados por él en la organización y financiamiento del proyecto de la Expedición Libertadora, sino igualmente respecto a decisiones que debió asumir cuando se produce, tan rápidamente, el desencuentro entre ambos países y ambas economías en la Guerra contra la Confederación de la década de 1830. Producto de ello, y a manera de una síntesis entre las colaboraciones O'Higgins - San Martín, en cuarto lugar se considera histórica e historiográficamente una especie de balance respecto a la imagen de O'Higgins a nivel peruano y latinoamericano, lo que igualmente requiere de observar no sólo los grandes proyectos 
pensados y soñados en conjunto con San Martín, sino igualmente parte de sus desavenencias las cuales no influyeron en los vaivenes que la figura de O'Higgins ha venido experimentando en los sitiales de la historia peruana, no por él mismo, sino por circunstancias que seguramente nunca fueron pensadas por ninguno de los dos héroes. Con todo, no deja de ser sensible el desenlace de las historias personales de ambos personajes, que seguramente buscaron la gloria y terminaron desentrañados de los territorios y sociedades que tanto imaginaron.

Una buena historia requiere igualmente de una buena enseñanza de la historia y sobre ello reflexiona el quinto de los trabajos del dossier. Si bien podemos coincidir en que la figura de O'Higgins ha sido utilizada por las dinámicas del Estado para fijar determinadas narrativas acerca de la nación, ello no significa afirmar que O’Higgins no tenga nada que aportar a la ciudadanía de hoy, incluso más allá de los intereses más inmediatos de quienes ocupan actualmente los cargos de poder en la sociedad. En ello, por cierto, la educación escolar ofrece un lugar adecuado, especialmente, dado el interés en las últimas décadas por acentuar más la formación de los futuros ciudadanos. Se trataría de que los propios estudiantes aprendan a deliberar el pasado buscando nuevas maneras de entender el presente y comprometiéndose con el futuro. En ese contexto, la figura de O'Higgins y la discusión en el aula respecto de su innegable papel como líder, puede aportar significativamente en la construcción de nuevos diálogos en torno a lo que significa ser ciudadano hoy.

Buena historia, buenas y adecuadas aproximaciones entre pasado y presente. Los héroes en su lugar, nosotros en la historia actual. ¿Qué nos pueden decir? No todo porque éste presente tiene sus propios elementos, preocupaciones y capacidades para decidir sobre sus futuros inmediatos. Pero, la historia no es sólo ruptura, sino también continuidades.

Eduardo Cavieres Figueroa 